mitochondria was examined in the endothelium in intact blood vessels. In controls, TRPV4 activation with GSK1016790A(GSK) generated repetitive Ca2+ oscillations that required $\mathrm{Ca} 2+$ influx. When the $\Delta \psi \mathrm{m}$ was depolarised, by the uncoupler carbonyl cyanide m-chlorophenyl hydrazine (CCCP) or the complex I inhibitor rotenone, TRPV4 activation generated a much larger $\mathrm{Ca} 2+$ rise and propagating multicellular $\mathrm{Ca} 2+$ waves. The ATP synthase inhibitor oligomycin did not potentiate TRPV4 mediated $\mathrm{Ca} 2+$ influx. GSK-evoked $\mathrm{Ca} 2+$ waves, that occurred when mitochondria were depolarised, persisted in a $\mathrm{Ca} 2+$ free extracellular solution i.e. were independent of $\mathrm{Ca} 2+$ influx. These signals were blocked by the TRPV4 channel blocker HC067047 (HC067), the SERCA inhibitor cyclopiazonic acid, the phospholipase C (PLC) blocker U73122 and the inositol triphosphate receptor (IP3R) blocker caffeine. These observations suggest that TRPV4 may directly activate $\mathrm{Ca} 2+$ release from the internal store. The large propagating waves were inhibited by the pannexin blocker probenecid and the extracellular ATP blockers suramin and apyrase. These results highlight a previously unknown role of mitochondria in shaping TRPV4 mediated $\mathrm{Ca} 2+$ signalling and show that TRPV4 may trigger ATP release via a pannexin hemichannel when mitochondria are depolarised.

Conflict of Interest $\mathrm{n} / \mathrm{a}$

\section{BS23 MRNA EXPRESSION PROFILING OF DUAL SPECIFICITY PHOSPHATASES (DUSPS) IN THE HYPERTENSIVE HEART}

${ }^{1}$ Zoe Haines, ${ }^{2}$ Josh Cull, ${ }^{1}$ Samuel Baldwin, ${ }^{1}$ Guy Whitley, ${ }^{2}$ Angela Clerk, 'Daniel Meijles. ${ }^{1}$ St George's University London, London, UK; ${ }^{2}$ University of Reading, UK

\subsection{6/heartjn-2021-BCS.221}

Introduction DUSPs dephosphorylate Ser/Thr and Tyr residues, most notably in the mitogen-activated protein kinases (MAPKs) that are activated by dual phosphorylation in a T-X-Y motif. Whereas some DUSPs preferentially dephosphorylate nuclear MAPKs (DUSP1/2/4/5), others dephosphorylate cytoplasmic MAPKs, including growth-promoting ERK1/2 (DUSP6/7/9) and stress-responsive p38-MAPKs or JNKs (DUSP8/10/16). MAPKs regulate cell growth/survival/death and are implicated in hypertensive heart disease. DUSPs may fine-tune these pathways and influence cardiac remodelling. Here, we assessed expression profiles of cardiac DUSP mRNAs in human heart failure and hypertensive rodents.

Methods Cardiac DUSP (DUSPs 1, 2, 4, 5, 6, 7, 8, 9, 10 and 16) mRNA expression was assessed in an archive of human left ventricular tissue from control $(n=12)$ and non-ischemic heart failure $(n=12)$ individuals. Rodent hypertension models were also studied: spontaneously hypertensive rats (SHRs; 1113 weeks, $\mathrm{n}=8$ ) were compared to age-matched WKY controls $(\mathrm{n}=8)$, and $\mathrm{C} 57 \mathrm{BL} / 6 \mathrm{~J}$ male mice $(10 \mathrm{wks})$ were infused with vehicle (acidified-PBS, $\mathrm{n}=16$ ) or $0.8 \mathrm{mg} / \mathrm{kg} / \mathrm{d}$ angiotensin II (AngII) for $24 \mathrm{~h}(\mathrm{n}=11)$ or $7 \mathrm{~d}(\mathrm{n}=12)$ using osmotic minipumps. In all studies, cardiac DUSP mRNA expression was assessed by $\mathrm{qPCR}$ relative to GAPDH. Statistical tests used a 1-way ANOVA with Holm-Sidak post-test (mouse studies) and unpaired t-tests (human and rat studies).

Results All DUSPs were detected in human hearts except DUSP5; DUSP7 was significantly suppressed (0.53 \pm 0.045 -fold, $\mathrm{p}=0.0065)$ in failing hearts. All DUSPs were detected in rat or mouse hearts. With chronic hypertension in SHRs, Dusp2
(1.39 \pm 0.10 -fold, $\quad \mathrm{p}=0.0090), \quad$ Dusp4 $\quad(1.90 \pm 0.17$-fold $\mathrm{p}=0.0004)$, Dusp6 (1.31 \pm 0.15 -fold, $\mathrm{p}=0.0190)$, Dusp7 (1.48 \pm 0.05 -fold, $\mathrm{p}<0.0001)$, Dusp8 $(2.01 \pm 0.15$-fold, $\mathrm{p}=0.0024)$ and Dusp16 $(1.22 \pm 0.05$-fold, $\mathrm{p}=0.0295)$ were all upregulated relative to WKY controls, whilst Dusp9 was decreased $(0.33$ \pm 0.08 -fold, $p=0.0232$ ). In contrast, in the acute model of murine hypertension with AngII-treatment, there were significant increases in Dusp8 (1.48 \pm 0.12 fold, $p=0.0003)$, Dusp10 (1.54 \pm 0.08 -fold, $\mathrm{p}<0.0001)$ and Dusp16 (1.37 \pm 0.06 -fold, $\mathrm{p}=0.0306)$ abundance at $24 \mathrm{~h}$, whilst only Dusp6 $(1.47 \pm 0.11$ fold, $p=0.0013$ ) was elevated at 7 days relative to vehicle controls.

Conclusion DUSPs are expressed in human and rodent hearts. With acute adaptation to hypertension, changes in DUSP profiles potentially modulate activities of stress-responsive MAPKs. However, in established hypertension and human heart failure, changes in DUSP profiles potentially modulate activities of cytoplasmic ERKs. Thus, DUSPs are likely to contribute to development of hypertensive heart disease.

Conflict of Interest None

\section{BS24 INVESTIGATING THE ROLE OF SALT-INDUCIBLE KINASE 2 (SIK2) IN HEART}

${ }^{1}$ Cecilia Facchi, ${ }^{1}$ Xin Wang, ${ }^{2}$ Elizabeth Cartwright, ${ }^{2}$ Delvac Oceandy. ${ }^{1}$ University of Manchester, Manchester, UK; ${ }^{2}$ Division of Cardiovascular Sciences, FBMH, University of Manchester

\subsection{6/heartjnl-2021-BCS.222}

Introduction Heart failure (HF) is characterised by an inadequate cardiac pumping ability and is one of the major causes of death worldwide. Numerous conditions lead to HF, such as myocardial infarction (MI). After MI, an extensive death of cardiac cells combined with limited regenerative capacity causes a pathological remodelling of the left ventricle, characterized by substantial fibrosis and hypertrophy. Replacement of damaged tissue or limitation of pathological progression are not achievable with current treatments. Thus, novel therapeutic approaches to address this unmet medical need are still required.

We decided to explore the unknown role of Salt-inducible kinase 2 (SIK2) in cardiac cells to potentially identify a novel target for HF therapy. Recently, this kinase has been identified as a key modulator of Hippo pathway-mediated stimulation of cell proliferation in Drosophila and a putative mediator of cardiac hypertrophy progression in response to chronic highsalt intake.

Methods and Results Firstly, we demonstrated that SIK2 protein is expressed in both fibroblasts and cardiomyocytes. We also identified a variation of its expression during the different developmental stages of the organism, displaying a greater level during the neonatal phase, and an elevated expression during HF progression, underlining the decisive importance of this kinase in myocardial tissue organization and remodelling.

We performed an in-silico analysis to unroll the mediators of this effect, which suggested that SIK2 activity is mainly mediated by either Hippo or Akt pathway. Exploiting an adenovirus overexpression system, we observed evidence of increased Akt phosphorylation levels. We also found that SIK2 overexpression in primary neonatal rat cardiomyocytes causes activation of LATS, one of the main components of the Hippo pathway, suggesting a potential SIK2-mediated regulation of the Hippo pathway. These alterations did not cause 
changes in survival rate or cell proliferation. However, it clearly promoted the induction of cell hypertrophy.

Conclusion In summary, these preliminary data suggest that SIK2 might modulate the hypertrophic response of cardiac tissue during pathological insults.

Conflict of Interest No

\section{BS25 TAM RECEPTOR AXL LOSS REGULATES SMOOTH MUSCLE CELL DIFFERENTIATION AND ACCELERATES ATHEROSCLEROSIS IN MICE}

'Lucy Mcshane, 'Danila Gurgone, ${ }^{1}$ Abdullatif Mohammed Bin-Khunayn, ${ }^{2}$ Eva Crespo, ${ }^{1}$ Gianluca Grassia, ${ }^{1}$ Neil MacRitchie, ${ }^{3}$ Mauro Giacca, ${ }^{4}$ Lorena Zentilin, ${ }^{5}$ Glykeria Karadimou, ${ }^{5}$ Gabrielle Paulsson-Berne, ${ }^{5}$ Peder S Olofsson, ${ }^{1}$ Thomas D Otto, ${ }^{2}$ Tomasz I Guzik, ${ }^{1}$ Mariola Kurowska-Stolarska, 'Pasquale Maffia. 'Centre for Immunobiology, Institute of Infection, Immunity and Inflammation, University of Glasgow, Glasgow, UK; ${ }^{2}$ Institute of Cardiovascular and Medical Sciences, University of Glasgow; ${ }^{3}$ School of Cardiovascular Medicine \& Sciences, King's College London British Heart Foundation Centre; ${ }^{4}$ Molecular Medicine Laboratory, International Centre for Genetic Engineering and Biotechnology (ICGEB): ${ }^{5}$ Center for Molecular Medicine, Karolinska Institutet

\subsection{6/heartjnl-2021-BCS.223}

Introduction The TAM receptors (Tyro3, Axl, and MerTK) are a distinct family of three receptor tyrosine kinases, namely Tyro3, Axl and MerTK, which play critical roles in cancer, inflammatory disorders and cardiovascular diseases. Axl, in particular, has been shown to influence multiple aspects of cardiovascular pathology via diverse effects on cells of both the vasculature and immune system through regulation of vascular remodelling, efferocytosis and inflammation. Clinical studies have shown that Axl is detectable in atherosclerotic plaques; however, the causal relationship between Axl and atherosclerosis is still uncertain, and results from mouse models fell short of defining the specific role(s) of Axl in the disease process.

Methods In order to quantify Axl expression in carotid endarterectomy atherosclerotic plaques we examined data from the Biobank of Karolinska Endarterectomy (BiKE). Using single-cell RNA sequencing (scRNA-seq) data from published atherosclerosis datasets we determined which cell types express Axl during pathology. Finally, we utilised an inducible atherosclerosis model in order to assess atherosclerosis formation in global Axl-deficient mice (Axl-/-). Female C57BL/6NQ (WT; $n=15)$ and Axl-/- $(n=21)$ mice were injected with $3 \times 1011$ vector genomes of AAV8-proprotein convertase subtilisin/kexin type 9 (PCSK9) and placed on a Western Diet (WD) for 12 weeks. Plaque size and percentage of necrotic core were determined in the aortic sinus using Oil Red O (ORO). Collagen content was determined using picrosirius red and polarised light microscopy. ScRNA-seq was performed to explore differences in Axl-/- vs. WT aortas at the cellular and molecular level.

Results We found expression of Axl in human carotid plaque to be significantly reduced in comparison to healthy control tissue $(\mathrm{P}=1.96 \mathrm{e}-06)$ in the BiKE cohort. Similarly, we detected less Axl RNA expression in the aortas of WDfed apolipoprotein-E-/- mice compared to WT $(\mathrm{P}<0.05)$. Analysis of published scRNA-seq databases found that Axl is expressed predominantly in the vascular smooth muscle cell (VSMC) compartment of the aortas in both healthy and atherosclerotic mice, with expression also observed in fibroblasts and macrophages. Global Axl-deficiency increased lesion size in the aortic sinus $(\mathrm{P}<0.001)$. While collagen content and necrotic core were not affected. ScRNA-seq on the aortas showed a switch versus a less contractile smooth muscle cell phenotype in Axl-/- mice compared to WT.

Conclusions In conclusion, our results indicate a protective role for Axl in atherosclerosis. The TAM receptor is reduced in diseased vessel compared to healthy controls in both human and mouse. Furthermore, global knock-out resulted in significantly increased plaque burden in mice. The necrotic core was not found to be influenced by Axl, suggesting that TAM receptor-mediated efferocytosis is not a key contributor to the role of Axl in atherosclerosis. Axl was found to be predominantly expressed in the VSMC compartment in the aortas of both healthy and diseased mice. Furthermore, Axl deficiency promoted VSMC phenotypic switching. These data support the hypothesis of a beneficial role of Axl in atherosclerosis via modulation of smooth muscle cell phenotype.

Conflict of Interest none

\section{BS26 THE PARTNERSHIPS IN CONGENITAL HEART DISEASE IN AFRICA STUDY (PROTEA): CLINICAL CHARACTERISTICS AND GENETIC FINDINGS FROM A SOUTH AFRICAN CONGENITAL HEART DISEASE COHORT}

${ }^{1}$ Timothy Spracklen, ${ }^{1}$ Thomas Aldersley, ${ }^{1}$ Nicole Saacks, ${ }^{1}$ Bianca de Koning ${ }^{1} J o h n$ Lawrenson, 'Paul Human, ${ }^{2}$ James Eales, ${ }^{1}$ Blanche Cupido, 'George Comitis, ${ }^{1}$ Rik De Decker, ${ }^{3}$ Barend Fourie, 'Lenise Swanson, ${ }^{1}$ Alexia Joachim, ${ }^{1}$ Phaphama Magadla, ${ }^{1}$ Malebogo Ngoepe, ${ }^{1}$ Liam Swanson, ${ }^{2}$ Alistair Revell, ${ }^{1}$ Raj Ramesar, ${ }^{1}$ Gasnat Shaboodien, ${ }^{1}$ Andre Brooks, ${ }^{1}$ Karen Sliwa, ${ }^{1} J o h n$ Anthony, ${ }^{1}$ Ayesha Osman, ${ }^{2}$ Bernard Keavney, 'Liesl Zuhlke. 'University of Cape Town, Cape Town, South Africa; ${ }^{2}$ University of Manchester; ${ }^{3}$ University of Stellenbosch

\subsection{6/heartjnl-2021-BCS.224}

Introduction Congenital heart disease (CHD) is the most common birth defect and a significant cause of paediatric morbidity and mortality worldwide. Epidemiological data from Africa are lacking, although this information is of importance in determining the burden of CHD and guiding policy. As a multifactorial disease, the role of genetic factors in $\mathrm{CHD}$ is increasingly recognised. However, the genetic contribution to CHD remains relatively unexplored in Africa. The Partnerships in CHD in Africa (PROTEA) project was established to better understand the epidemiology and genetics of CHD in subSaharan Africa. The aim of this investigation is to describe the clinical and genetic characteristics of a cohort of CHD patients from the Western Cape, South Africa.

Methods PROTEA is a multicentre, prospective registry of CHD patients, recruited from seven hospitals in the Western Cape, South Africa. Patients with any structural CHD were eligible for inclusion, this excluded patients with isolated patent foramen ovale, peripheral pulmonary stenosis or patent ductus arteriosus in premature infants. Some of these patients were consented into the genetics study, for which a DNA biorepository was established. These patients were investigated using exome sequencing and/or chromosomal microarray (CMA) to identify disease-causing mutations or copy number variants in established CHD genes.

Results A total of 1,473 participants were recruited into the PROTEA registry between April 2017 and March 2019 (median age 1.9 years, 51\% male). Compared to international cohorts, ventricular (PR: 1.8, 95\%CI: 1.63-1.97) and atrial (PR: 1.4, 95\%CI: 1.20-1.57) septal defects were significantly 